Proceedings of the 2012 Winter Simulation Conference

C. Laroque, J. Himmelspach, R. Pasupathy, O. Rose, and A. M. Uhrmacher, eds.

\title{
A SURVEY ON THE USE OF SIMULATION IN GERMAN HEALTHCARE
}

\author{
Patrick Kirchhof \\ BearingPoint $\mathrm{GmbH}$ \\ Gladbecker Straße 5 \\ D-40472 Düsseldorf, GERMANY
}

\author{
Nicolas Meseth \\ Deloitte Consulting GmbH \\ Schwannstraße 6 \\ D-40476 Düsseldorf, GERMANY
}

\begin{abstract}
This paper reports on the results of a survey conducted among German healthcare institutions to collect data about the use of simulation in the respective field. The setup follows a survey published in Greasley (2008). One goal of the survey was to assess how many institutions have used simulation as a decision making tool before, and if they plan to do so in the future. Another focus are the potential reasons against the use of simulation, which are grouped into the categories costs, awareness, skills \& experience, organizational-, and technical obstacles. The results indicate that while the use of simulation in German healthcare is low, costs are the main reason against a wider adoption. Following are lack of awareness among decision makers, and a lack of skills within the internal staff. An alleged negative reputation of simulation could not be confirmed.
\end{abstract}

\section{INTRODUCTION}

Simulation has been used to assist decision making in the field of healthcare for many years. But while the academic literature in this domain is massive and increases constantly, records of applications to real-life problems remain rare (Brailsford et al. 2009). Several recent literature reviews present systematic assessments of academic contributions, analyzing the extent of simulation applications to real-life problems. For example, Jun, Jacobson, and Swisher (1999) survey the literature regarding applications of discreteevent simulation modeling to healthcare clinics. Fone et al. (2003) present an extensive review of 182 papers on the use of simulation in healthcare. Brailsford et al. (2009) analyze academic literature on simulation and modeling in healthcare unrestricted to a specific domain of application in order to determine the relative frequency of use of operational research modeling approaches in healthcare, their respective fields of application, and the level of implementation. In a recent literature review Sobolev, Sanchez, and Vasilakis (2011) analyze the use of simulation for modeling patient flow.

Despite the large number of academic publications, the practical applications of simulation in healthcare are relatively scarce, especially compared to sectors like manufacturing or logistics. Jahangirian et al. (2012) present a cross-sectoral study in which they compare academic work in these domains. They find that only $8.0 \%$ of the articles on simulation in healthcare report about a real problem, compared to $48.9 \%$ in manufacturing and business. In a similar investigation, Eldabi (2009) classifies papers on applications of simulation in healthcare, defense / aerospace, and industry / business into three categories. The first category contains those papers reporting on a real problem with real stakeholders. The second one includes articles that addressed real-life problems without engagement form real stakeholders. All entirely theoretical papers are in the third category. Figure 1 shows his findings. It clearly illustrates the difference between these sectors, and underlines the prevalence of theoretical work in contrast to practical applications in healthcare simulation.

Although the debate on the barriers to implementation of simulation in the healthcare sector is long standing and is still carried on vividly (Eldabi 2009), the reasons for the modest adoption of simulation in this field are not clear (Brailsford et al. 2009), and many possible explanations are discussed. Fackler 


\section{Kirchhof and Meseth}

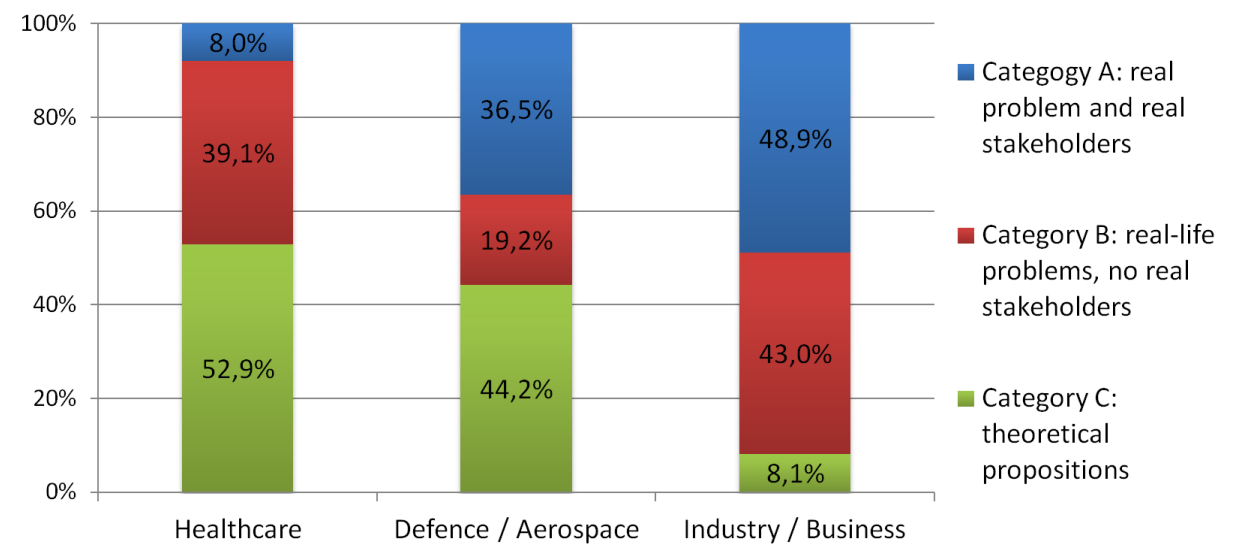

Figure 1: Cross-sectoral comparison of practical simulation applications based on Eldabi (2009).

and Speader (2011) argue that the required qualifications for simulation repel clinical practitioners, who are not trained accordingly. In line with that, Jahangirian, Taylor, and Young (2010) see the absence of familiarity with the simulation concepts among practitioners as one of the major challenges. More technical, Virtue, Kelly, and Chaussalet (2011) report from their experience that stakeholder desire simplified models with shorter development cycles. Eldabi et al. (2010) see the greatest barrier to more intensive use in a mismatch between the healthcare modeling theory and the practical requirements of practitioners. Jahangirian et al. (2012) identify the typically large organization found in hospitals as an important reason, as large organizations tend to resist stronger to change. In addition, Eldabi (2009) presents an overview of further reasons mentioned in literature, such as the resistance to the unfamiliar, the dehumanizing nature of simulation, the culture of mistrust of industrially based methods, the cost of modeling, poor data availability, conflicts of interest and the lack of uniformity among healthcare facilities.

As plausible as these arguments are, they are not empirically verified, but are rather a result of the researchers' logical reasoning about their personal practical experience. The intention of this paper is to contribute to a better understanding of the existing barriers to more widespread adoption of simulation and the relative importance of these reasons through an empirical assessment. To achieve this goal, an empirical survey was conducted in which healthcare institutions were asked about their prior experiences with simulation and their reasons for not incorporating it into their decision making and planning processes.

The results of the survey suggest that not all of the aforementioned reasons are equally relevant, at least not in the German healthcare institutions, to which this examination is limited. It was found that one barrier is a very fundamental one. Practitioners are to a large extent not aware of the benefits of simulation as a method to assist decision making or planning processes. To many of them it is not clear how or when to apply simulation, and they do not consider it as an option from the first. This lack of basic understanding and the lack of experiences appear to be major reasons why simulation is not as strongly embraced by the healthcare sector as it is by others. The costs associated with conducting a simulation project are another major reason. Both, initial costs and costs for model development and data acquisition appear to be significant obstacles. As a consequence of a relative low use of simulation and the lack of experience, the skills among internal staff are insufficient for simulation modeling and statistical analysis. In contrast, organizational and technical factors seem less relevant.

Our paper is structured as follows. In Section 2 the design and the sample of the survey are portrayed. The characteristics of the participating institutions are described in Section 3. In Section 4 the survey results regarding the prior use of simulation and the planned use in the future are presented. The fifth section contains the analysis of the obstacles to wider use of simulation, and Section 6 concludes the paper. 


\section{Kirchhof and Meseth}

\section{THE SURVEY}

\subsection{Design of the Survey}

The design of the survey and the questionnaire follows the study conducted by Greasley (2008), who analyzes the use of simulation in UK companies across various industrial sectors. The authors made adjustments and extensions only to better fit the healthcare sector.

To ensure the correct understanding of the term simulation in the context of the survey, it was clearly indicated that the survey refers to process simulation and not training simulation or else. This is especially important since training simulation is extensively used in healthcare to train staff in certain procedures, and therefore exists a high risk that the term is misunderstood.

The questionnaire consists of three parts. The first contains both closed and semi-closed questions about the institution itself, its size (e.g., number of employees, cases per year), and its type of funding (e.g., public, private, nonprofit).

The second section addresses the previous use of simulation. It is asked if simulation had been used before and whether plans of future use exist. In case simulation has already been used, the survey asks more detailed questions regarding the kind of simulation projects, the software in use, and the organization of the projects (e.g., internal employees or external consulting), and the attitude towards future use of simulation.

The structure of the third section is largely adopted from Greasley (2008). In order to identify the main obstacles of simulation in German hospitals, the participants rated the importance of pre-formulated reasons why in their institution simulation is not used, or if it has been used, why it is not used more often. In total, the survey presents 22 reasons divided in the categories cost, awareness, skills, organizational, and technical limitations. The response options lie on a Likert scale with numbers from 1 (not a reason) to 5 (major reason). Unlike Greasley's original study, there is no clear distinction between users and non-users of simulation, as prior to the survey the number of users was assumed to be small.

\subsection{Survey Sample}

The participating hospitals were randomly chosen from a directory of German health institutions disregarding their specific characteristics. Only dedicated psychiatric and rehabilitation clinics were excluded from the sample under the assumption that their processes, structures, and requirements are different from those of regular hospitals of general care.

In order to increase the response rate, the clinics were contacted by telephone and informed about the study. They were asked about their willingness to participate, and those who rejected were excluded from the sample. The questionnaire of this survey was then sent to 121 German hospitals of which 32 responded, which leads to a response rate of $26.4 \%$.

To identify the best qualified person in the hospital to fill out the survey, the authors asked the receptionist on the phone for the contact who is either in charge of operations, controlling, or information technology. The authors then asked the referred person if he thought he was the person that should know if simulation was used in the hospital. If not, the authors asked to name the right contact. Since the survey did not require to include the name of the answerer, it is possible that another person than the contacted person filled out the survey.

\section{CHARACTERISTICS OF PARTICIPATING INSTITUTIONS}

Figure 2 shows the characteristics of the participating institutions. Of the responding hospitals, $22 \%$ were privately owned, $34 \%$ were public institutions and $44 \%$ were nonprofit institutions. This roughly reflects the current distribution of funding types (32.9\% private, $36.6 \%$ nonprofit, $30.5 \%$ public) presented by the German Federal Statistical Office (Statistisches Bundesamt 2010). 


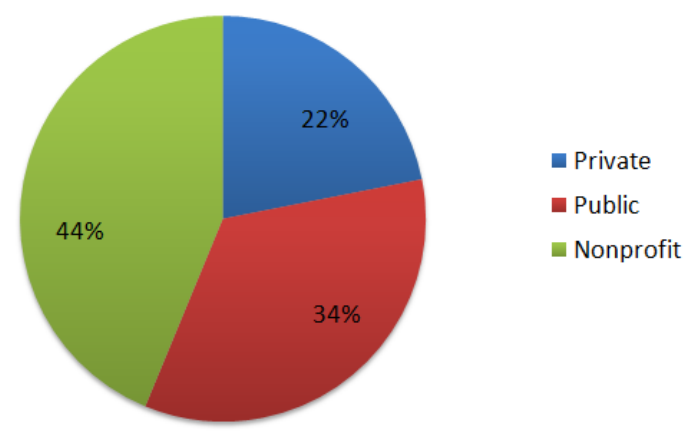

Figure 2: Funding types of the participating institutions.

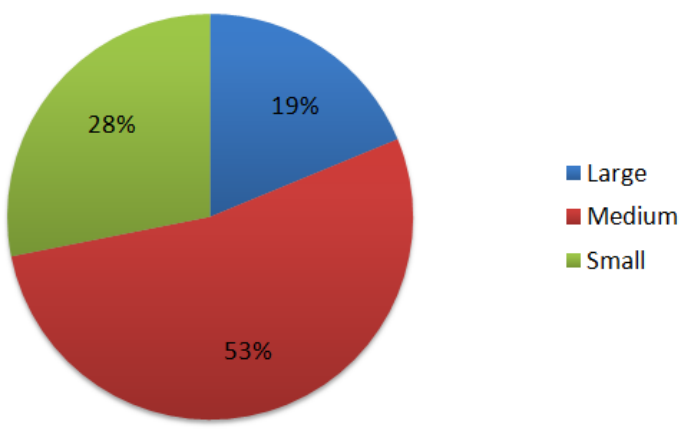

Figure 3: Size of the participating institutions.

The size of the institutions is measured by the number of patient beds, the number of cases per year, and the number of employees. The values of these three measures were aggregated by introducing the categories of large, medium, and small institutions. The latter have less than 400 employees, less than 190 beds, and less than 7.500 cases. Large institutions have more than 1.200 employees, more than 600 beds, and more than 23.000 cases per year. The values for medium sized institutions are in between these ranges. According to this categorization, the sample contains $28 \%$ small, $53 \%$ medium sized, and $19 \%$ large institutions, as shown in Figure 3.

\section{ANALYSIS OF PRIOR EXPERIENCES AND PLANNED USE OF SIMULATION}

\subsection{Prior Experiences}

The vast majority $(81.2 \%)$ of the participating institutions has no prior experience with any sort of simulation. Only $18.8 \%$ state that in their institution simulation has been used before, where $6.3 \%$ are rarely using it, $9.4 \%$ are doing so occasionally, and only $3.1 \%$ report a frequent use as shown in Figure 4.

Figure 5 indicates that the use of simulation correlates with the size of the institution. All of the small institutions answered that they never used simulation before. Of the medium sized institutions, $17.7 \%$ replied they use simulation rarely or occasionally, whereas an extensive use of simulation was stated by the group of the large institutions. Here, even $50.0 \%$ claim to use simulation occasionally or often. The correlation coefficient between the measures of size and the prior use of simulation is $r=0.52$.

One might assume that privately operated institutions use simulation to a greater extent than publicly or charitably funded ones, due to higher economical pressure and a more pronounced business rationale. This 


\section{Kirchhof and Meseth}

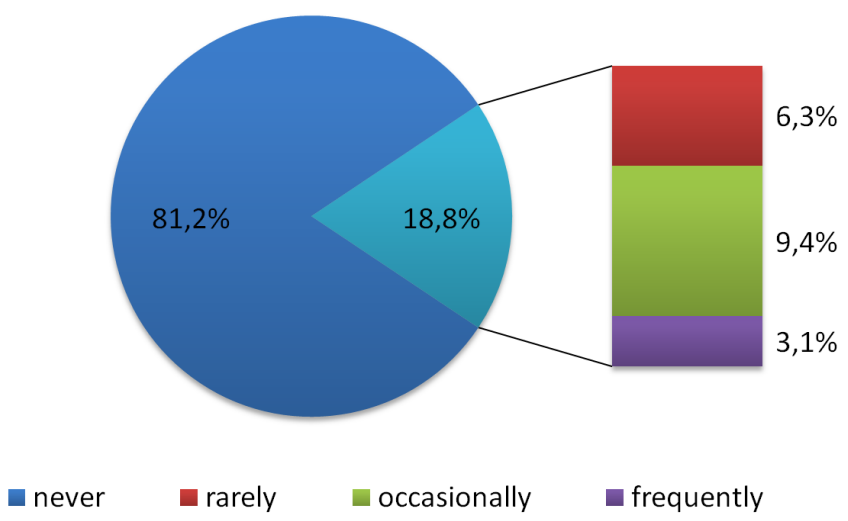

Figure 4: Prior use of simulation.

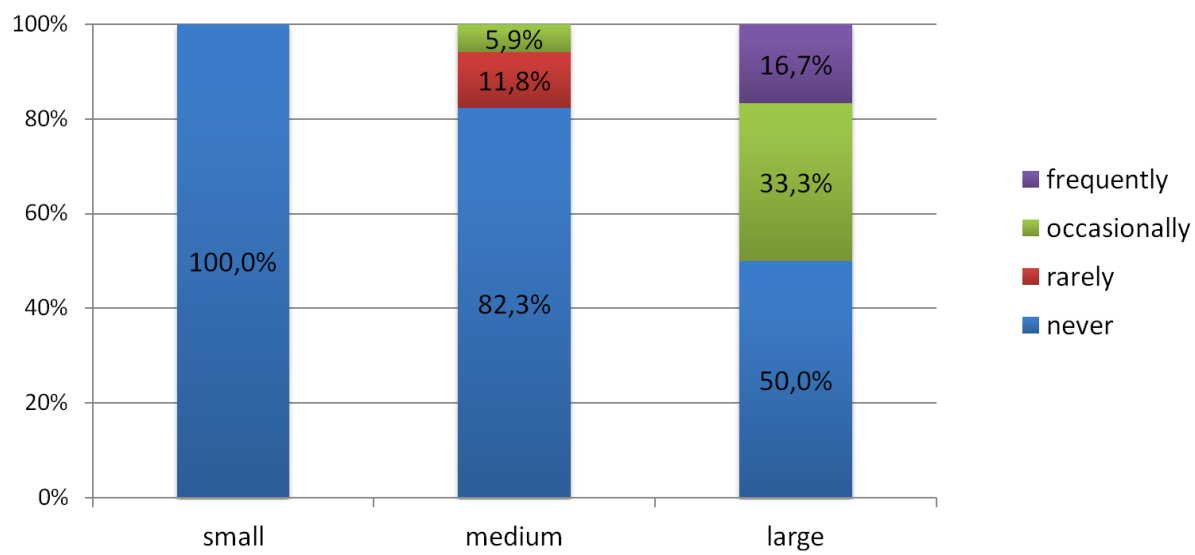

Figure 5: Prior use of simulation depending on the institution's size.

hypothesis cannot be confirmed by the survey's data. In fact, public institutions contain the highest ratio of simulation users $(36.4 \%)$, whereas only $7.1 \%$ of the nonprofit, and $14.3 \%$ of the private institutions previously employed simulation. The correlation coefficient between the funding type and the measure of prior use of simulation is $r=0.30$. The data suggests that the size of the institution is more relevevant for the extent of simulation use than the type of the funding.

\subsection{Planned Use}

The question whether the use of simulation is planned in the future was negated by $77.4 \%$ of the participants. $16.1 \%$ stated that they would generally endorse a simulation project, but that there were no immediate plans. Only $6.5 \%$ had projects concretely planned for the future.

Again, the planned use of simulation is more pronounced among the larger institutions, as shown in Figure 6. Only $11.0 \%$ of the small ones would generally endorse a simulation project, and none of them indicated concrete plans for future simulation use. Of the medium sized institutions, $6.2 \%$ had concrete plans and $18.8 \%$ indicated a general acceptance. The largest extent of planned use was measured among large institutions. Here, $16.7 \%$ would generally endorse a simulation project, and another $16.7 \%$ reported projects being planned. 


\section{Kirchhof and Meseth}

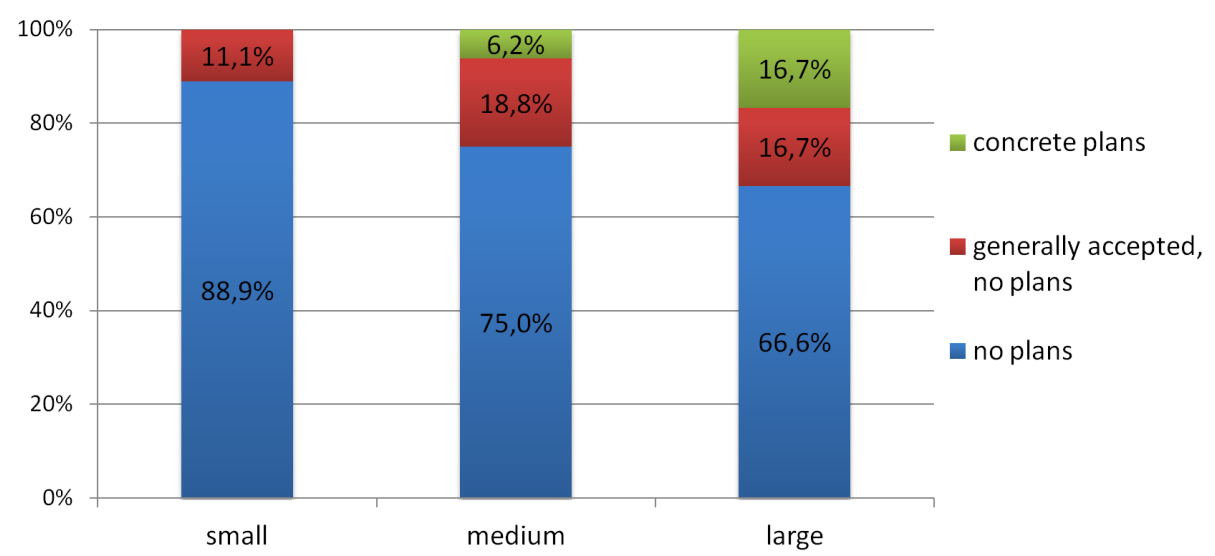

Figure 6: Planned use of simulation depending on the institution's size.

\section{ANALYSIS OF OBSTACLES FOR THE USE OF SIMULATION}

\subsection{Overview}

Figure 7 gives an overview of the survey results for possible obstacles to a wider use of simulation in healthcare institutions. The questions are grouped into the five categories costs, simulation awareness, skills and experience, organizational factors, and technical factors. The table displays the percentages by which the given reasons were graded by the respondents. Following Greasley (2008), possible answers lie on a Likert scale from one to five, five meaning a strong agreement, one meaning the mentioned reason is irrelevant. In Figure 7, answer 1 and 2 were combined into the group Not a reason and 4 and 5 into Major reason. All responses 3 were discarded in this table.

\subsection{Costs}

The results strongly suggest that costs are a major reason against the use of simulation in healthcare. At least $63.3 \%$ or more of the respondents rate any of the four reasons (Q1-Q4) as major. In accordance, a maximum of $10.0 \%$ thinks costs are not a reason. Regarding costs, there is no significant difference in the answers between the different institution sizes or funding types.

More insights could be gained from querying which types of costs (software \& hardware, training, data collection, consultancy) is seen as the largest component in the whole simulation study. This information is not contained in the current survey.

\subsection{Simulation Awareness}

Only $20.0 \%$ see no reason in the lack of knowledge where simulation can be applied, while a rough quarter $(26.7 \%)$ believes it is a major reason (Q5). While the respondents seem to be aware of where simulation can be applied, $66.7 \%$ state that a missing awareness about the benefits of simulation is a major reason (Q6). This could mean that decision makers know they could develop a simulation model for their problem, but they do not know how this would help them, or what questions to ask the model. Consequently, these potential simulation users have no incentive to invest in simulation and to explore its benefits as a decision making tool.

Another interesting fact is that only a total of $16.7 \%$ of the respondents have reports of negative experiences from others. An alleged negative reputation of simulation as a decision making tool is therefore not confirmed by the survey results. 


\section{Kirchhof and Meseth}

\begin{tabular}{|c|c|c|c|}
\hline $\mathbf{Q}$ & Reasons for non use / obstacles & Not a reason & Major reason \\
\hline & \multicolumn{3}{|l|}{ Costs } \\
\hline 1 & Initial Costs for software, hardware \& training & $6,7 \%$ & $76,7 \%$ \\
\hline 2 & Costs for model development & $6,7 \%$ & $63,3 \%$ \\
\hline 3 & Costs for data collection & $10,0 \%$ & $66,7 \%$ \\
\hline 4 & Costs for external consultancy & $6,7 \%$ & $76,7 \%$ \\
\hline & \multicolumn{3}{|l|}{ Simulation Awareness } \\
\hline 5 & Lack of knowledge where simulation can be applied & $20,0 \%$ & $26,7 \%$ \\
\hline 6 & Lack of knowledge of benefits of simulation & $6,7 \%$ & $66,7 \%$ \\
\hline 7 & Assumption that simulation is not suitable for problem & $23,3 \%$ & $46,7 \%$ \\
\hline 8 & Heard of negative experiences from others & $73,3 \%$ & $16,7 \%$ \\
\hline & \multicolumn{3}{|l|}{ Skills and Experience } \\
\hline 9 & Lack of staff skills in simulation development & $3,3 \%$ & $80,0 \%$ \\
\hline 10 & Lack of staff skills for statistical analysis & $16,7 \%$ & $60,0 \%$ \\
\hline 11 & Negative experiences in past use of simulation & $75,0 \%$ & $16,7 \%$ \\
\hline & \multicolumn{3}{|l|}{ Organizational } \\
\hline 12 & Low confidence that results of simulation will lead to change & $26,7 \%$ & $30,0 \%$ \\
\hline 13 & Fear of loss of decision sovereignty through simulation & $70,0 \%$ & $6,7 \%$ \\
\hline 14 & Intuitive (non analytical) approach to decision making preferred & $23,3 \%$ & $40,0 \%$ \\
\hline \multirow[t]{2}{*}{15} & Lack of confidence in simulation & $46,7 \%$ & $26,7 \%$ \\
\hline & \multicolumn{3}{|l|}{ Technical Limitations } \\
\hline 16 & Other techniques provide sufficient information & $23,3 \%$ & $43,3 \%$ \\
\hline 17 & Time to develop simulation is too slow for decision making & $6,7 \%$ & $60,0 \%$ \\
\hline 18 & Lack of flexibility in reusing simulation models & $26,7 \%$ & $16,7 \%$ \\
\hline 19 & Simulation model requires too much maintenance & $6,7 \%$ & $53,3 \%$ \\
\hline 20 & Assumptions in modeling make results unrealistic & $30,0 \%$ & $30,0 \%$ \\
\hline 21 & Lack of connectivity of simulation with databases $\&$ other IT & $26,7 \%$ & $40,0 \%$ \\
\hline 22 & Components of a simulation model cannot be reused & $26,7 \%$ & $20,0 \%$ \\
\hline
\end{tabular}

Figure 7: Overview of the reasons for not using simulation or not using it to a greater extent.

As with costs, the responses in this category showed no sensitivity to the institution's size or funding type either.

\subsection{Skills and Experience}

The data suggests that missing skills are a problem in healthcare institutions. Four out of five (80.0\%) state that they see a lack of simulation skills in their institution as a major reason (Q9). Among small institutions it is even $100.0 \%$. This conforms with the $76.7 \%$ who see the costs for external consultancy as a major reason $(\mathrm{Q} 4)$, as external help would be necessary to compensate the lack of simulation skills among the internal staff. The data draws a similar picture with regard to a lack of statistical skills: $60.0 \%$ see this as a major reason $(\mathrm{Q} 10)$.

Only $16.7 \%$ indicated negative experiences as a major reason, while $75.0 \%$ said this was not a reason (Q11). This compares well with the result that only $16.7 \%$ named reports of negative experiences from others as a major reason, while for $73.3 \%$ this was not a reason. 


\section{Kirchhof and Meseth}

\subsection{Organizational Obstacles}

In the overall results, $30.0 \%$ see a low confidence in the simulation results actually leading to a change as a major reason against its use (Q12). A distinction of the answers to this question by the size of the institution reveals that this opinion is predominant in small institutions (50.0\%), and absent in large ones $(0.0 \%, 31.3 \%$ in medium sized institutions). This is remarkable, as the intuitive answer would suggest that change is easier accomplished in smaller organizations, because structures are less static and one expects less resistance. The results also contrast Jahangirian et al. (2012), who point out that hospitals are usually very large organizations and that this might be among the main problems for the adoption of simulation in healthcare.

There seems to be a consensus among all respondents that intuitive, non-analytical approaches are favored instead of simulation ( $40.0 \%$ versus $23.3 . \%$ ). On the other hand, with some probability, the reason is not a lack of confidence in simulation per se, as only $26.7 \%$ marked this a major reason (Q15). This could indicate that decision makers believe that simulation could solve their problem, but other reasons (e. g. costs) prevent them from using it.

In total, the data suggests that organizational issues are not as substantial than other categories, such as the costs of simulation.

\subsection{Technical Obstacles}

According to the survey results, simulation bears a certain overhead that restrains decision makers from investing in it. $60.0 \%$ see a major reason in the fact that the development of simulation models is too slow for the decision making process (Q17). Moreover, the majority (53.3\%) thinks that a simulation model requires too much maintenance to be used for decision making (Q19). Further, $40.0 \%$ believe that simulation cannot be well integrated with existing databases and IT systems (Q21), while only $26.7 \%$ say this is not a reason. In addition, a number of decision makers (43.3\%) seems to favor other, maybe less ponderous methods, to satisfy their information needs (Q16). This matches with Q14, where $40.0 \%$ stated that more intuitive approaches are preferred for decision making.

\section{CONCLUSION}

In summary, the results permit the following conclusions:

- The use of simulation in German healthcare is low compared to other fields, and there seems to be a correlation between the size of the institution and the probability of simulation use (past and future). Further, there seems to be no connection between the funding type and the probability of simulation use.

- Costs are a major reason against use of simulation for institutions of any size or funding type. Moreover, a lack of awareness of the benefits plays a major role. Lack of skills for simulation development and statistical analysis seem to be another reason that could be a consequence of the low use and lack of experience. Organizational issues seem to have little impact. Considering technical aspects, the main problem is that simulation carries a certain overhead, and faster and more intuitive methods are available.

- There seems to be no negative reputation of simulation among German healthcare institutions.

One goal of the survey was to examine the past and future use of simulation in German healthcare institutions. In the sample of $n=32$ respondents, only $18.8 \%$ have used simulation in the past, and only $3.1 \%$ use it on a regular basis. The data shows a correlation between the size of the institution and the probability that simulation is in use: among large institutions, $50.0 \%$ claim they use simulation occasionally or even often, while in small ones there is no use reported at all. The data does not allow any conclusion with regard to the funding type of the institution and the probability that simulation is used. 


\section{Kirchhof and Meseth}

A similar dependency on the size of the institution was found for the planned use of simulation. In the sample, $16.1 \%$ are considering simulation for the future without concrete plans, and another $6.5 \%$ already have immediate plans. When considering only large institutions, the share with immediate plans is $16.7 \%$ (small: $0.0 \%$, medium: $6.2 \%$ ).

Examining the obstacles for the use of simulation, costs and lack of skills are seen as the most severe ones to overcome. Roughly 3 out of 4 respondents rated any of the reasons related to costs as major ones. 4 out of 5 say that a lack of skills among their staff is a problem, and 3 out of 5 testify the same for a lack of statistical skills. Two thirds believe that a major reason is unawareness of the benefits from simulation. On the other hand, organizational reasons seem to constitute no obstacle. Counterintuitively, there seems to be no concern that simulation will not lead to change among large institutions, while $50.0 \%$ of the small institutions see this as a major reason. The results indicate that simulation is seen as causing too much overhead, as $60.0 \%$ believe that simulation development is too slow for decision making, $53.3 \%$ answered that a simulation model is too maintenance-dependent, and $40.0 \%$ see a problem with integrating simulation with databases and IT.

With this survey, a first step was taken to empirically study the reasons why simulation is not as much embraced in healthcare as it is in other disciplines such as manufacturing, logistics, or military. While this work focuses on German healthcare institutions, it would be insightful to compare its results to those of surveys from different countries in order to analyze the relevance of cultural aspects and the structural differences in national healthcare systems. Moreover, the results should be checked against similar surveys from other disciplines to identify similarities as well as differences between them.

When interpreting the results, one must take into account the following possible limitations. Although the survey was designed with care to avoid misunderstandings about the questions or how the term simulation is defined in this context, the risk cannot be completely ruled out. Furthermore, it cannot be guaranteed that the best qualified person in the institution filled out the survey, although each receiving institution was interviewed for the best contact prior to sending out the survey. Regarding sample size, the authors obtained a satisfactory response rate of $26.4 \%$, but this evaluates to an absolute number of only 32 responses. The results would be more expressive if more responses could be included.

\section{REFERENCES}

Brailsford, S. C., T. Bolt, C. Connell, J. H. Klein, and B. Patel. 2009, December. "Stakeholder engagement in health care simulation". In Proceedings of the 2009 Winter Simulation Conference, edited by M. D. Rossetti, R. R. Hill, B. Johansson, A. Dunkin, and R. G. Ingalls, 1840-1848. Piscataway, New Jersey: Institute of Electrical and Electronics Engineers, Inc.

Brailsford, S. C., P. R. Harper, B. Patel, and M. Pitt. 2009. "An analysis of the academic literature on simulation and modelling in health care". Journal of Simulation 3:130-140.

Eldabi, T. 2009, December. "Implementation issues of modeling healthcare problems: misconceptions and lessons". In Proceedings of the 2009 Winter Simulation Conference, edited by M. D. Rossetti, R. R. Hill, B. Johansson, A. Dunkin, and R. G. Ingalls, 1831-1839. Piscataway, New Jersey: Institute of Electrical and Electronics Engineers, Inc.

Eldabi, T., G. T. Jun, J. Clarkson, C. Connell, and J. H. Klein. 2010, December. "Model driven healthcare: disconnected practices". In Proceedings of the 2010 Winter Simulation Conference, edited by B. Johansson, S. Jain, J. Montoya-Torres, J. Hugan, and E. Yücesan, 2271-2282. Piscataway, New Jersey: Institute of Electrical and Electronics Engineers, Inc.

Fackler, J., and M. Speader. 2011, December. "Why doesn't Heealthcare embrace Simulation and Modeling? What would it take?". In Proceedings of the 2011 Winter Simulation Conference, edited by S. Jain, R. R. Creasey, J. Himmelspach, K. P. White, and M. Fu, 1137-1142. Piscataway, New Jersey: Institute of Electrical and Electronics Engineers, Inc. 


\section{Kirchhof and Meseth}

Fone, D., S. Hollinghurst, M. Temple, A. Round, N. Lester, A. Weightman, K. Roberts, E. Coyle, G. Bevan, and S. Palmer. 2003. "Systematic review of the use and value of computer simulation modelling in population health and health care delivery". Journal of Public Health 25 (4): 325-335.

Greasley, A. 2008. Enabling a Simulation Capability in the Organisation. London: Springer.

Jahangirian, M., A. Naseer, L. Stergioulas, T. Young, T. Eldabi, S. Brailsford, B. Patel, and P. Harper. 2012. "Simulation in health-care: lessons from other sectors". Operational Research 12:45-55.

Jahangirian, M., S. J. E. Taylor, and T. Young. 2010, December. "Economics of modeling and simulation: reflections and implications for healthcare". In Proceedings of the 2010 Winter Simulation Conference, edited by B. Johansson, S. Jain, J. Montoya-Torres, J. Hugan, and E. Yücesan, 2283-2292. Piscataway, New Jersey: Institute of Electrical and Electronics Engineers, Inc.

Jun, J., S. Jacobson, and J. Swisher. 1999. "Application of discrete-event simulation in health care clinics: A survey". The Journal of the Operational Research Society 50 (2): 109-123.

Sobolev, B., V. Sanchez, and C. Vasilakis. 2011. "Systematic Review of the Use of Computer Simulation Modeling of Patient Flow in Surgical Care". Journal of Medical Systems 35:1-16.

Statistisches Bundesamt 2010. "Krankenhäuser: Eckdaten der Krankenhäuser 2010".

Virtue, A., J. Kelly, and T. Chaussalet. 2011, December. "Using simplified discrete-event simulation models for health applications". In Proceedings of the 2011 Winter Simulation Conference, edited by S. Jain, R. R. Creasey, J. Himmelspach, K. P. White, and M. Fu, 1154-1165. Piscataway, New Jersey: Institute of Electrical and Electronics Engineers, Inc.

\section{AUTHOR BIOGRAPHIES}

PATRICK KIRCHHOF studied business at the University of Osnabrueck, Germany. From 2007 to 2010 he was research assistant and doctoral student at the department for operations management and information systems. Since then, he works as a management consultant in the field of supply chain management, mainly in the automobile industry. In 2012 he received his doctorate for his work on the comparison of planning methods using component based simulation. His email address is Patrick.Kirchhof@BearingPoint.com.

NICOLAS MESETH studied Information Systems at the University of Osnabrueck, Germany. From 2006 to 2009 he was a research assistant and doctoral student at the department for operations management and information systems. Since then, he works as an IT-consultant in Germany with a focus on Business Intelligence. He received his doctorate in 2011. His email address is nmeseth@Deloitte.de. 